\title{
Does Rectus Sheath Block Analgesia Alter Levels of the Oxidative Stress Biomarker Glutathione Peroxidase: A Randomised Trial of Patients with Cancer and Benign Disease
}

\author{
MARTIN PURDY ${ }^{1}$, JARI KÄRKKÄINEN ${ }^{1}$, MERJA KOKKI ${ }^{2}$, MAARIT ANTTILA ${ }^{3}$, SAMULI ASPINEN ${ }^{1}$, \\ PETRI JUVONEN $^{1}$, HANNU KOKKI $^{2}$, KARI PULKKI $^{4,5}$, TUOMO RANTANEN $^{1}$ and MATTI ESKELINEN ${ }^{1}$ \\ Departments of ${ }^{1}$ Surgery, and ${ }^{2}$ Anaesthesia and Operative Services, \\ Kuopio University Hospital and School of Medicine, University of Eastern Finland, Kuopio, Finland; \\ ${ }^{3}$ Department of Gynaecology, Kuopio University Hospital, Kuopio, Finland; \\ ${ }^{4}$ Department of Clinical Chemistry, School of Medicine, University of Eastern Finland, and \\ ${ }^{5}$ Eastern Finland Laboratory Centre, Kuopio, Finland
}

\begin{abstract}
Aim: To evaluate whether the overall satisfaction, as measured by numeric rating scale (NRS), regarding rectus sheath block (RSB) analgesia is associated with the plasma glutathione peroxidase (GPXI) level. The second end-point of the study was to evaluate the differences in GPX1 levels in patients with and without RSB analgesia, with special emphasis on benign or malign disease status. Patients and Methods: Initially, 56 patients were randomized to the placebo group $(n=12)$ and to one of three active $R S B$ analgesia groups: single-dose $(n=16)$, repeated-dose $(n=12)$ and continuous infusion $(n=16)$ groups. The plasma level of GPXI was measured at three time points: just before, immediately after and $24 h$ after surgery. The overall satisfaction and an opinion on the success of the analgesic procedure were surveyed using an 11-point numeric rating scale $24 h$ postoperatively (NRS from 0, completely dissatisfied, to 10, fully satisfied). Results: The placebo group and the three active groups were similar in terms of their perioperative data. The plasma level of GPXI decreased postoperatively in all four groups. No differences were detected in the GPXI values between the placebo and the three active groups combined preoperatively and immediately after operation. However, the patients in the single-dose group had a significantly lower median GPXI values $24 \mathrm{~h}$ after surgery compared to the three other groups
\end{abstract}

Correspondence to: Matti Eskelinen, MD, Ph.D., School of Medicine, University of Eastern Finland, P.O. Box 100, FI-70029 KYS, Finland. Tel: +358 17173311, Fax: +358 17172611, GSM: +358 400969444, e-mail: matti.eskelinen@kuh.fi

Key Words: Cancer, oxidative stress, glutathione peroxidase, analgesia. separately $(p=0.032)$. The median (interquartile range) plasma level of GPXI differed significantly between patients with benign disease and those with cancer preoperatively (18.0, 12.5-22.0 versus 10.0, 6.3-18.8 pg/ml, $p=0.006)$ and cancer diagnosis was correlated with lower individual plasma GPX1 values ( $r=-0.42, p=0.004)$. Conclusion: The placement of RSB analgesia does not significantly affect the level of oxidative stress biomarker GPXI in patients with benign disease or cancer. A new finding with possible clinical relevance is that patients with cancer appeared to have a trend for lower plasma GPX1 values.

Glutathione peroxidase (EC 1.11.1.9; GPX) is the general name of an enzyme family with peroxidase activity whose main biological role is to protect organisms from oxidative damage by free oxygen radicals and generation of reactive oxygen species (ROS) (1-3). GPX reduces lipid hydroperoxides to their corresponding alcohols and free hydrogen peroxide to water. It has been shown that a low plasma GPX concentration in patients with type 2 diabetes correlates with macroalbuminuria and the stage of diabetic nephropathy (4). In one study, the activity of GPX was lower in patients with relapsing-remitting multiple sclerosis (5), and Katar et al. suggested that GPX plays a role in the development of celiac disease (6).

Abdominal surgery is associated with an inflammatory reaction and generation of oxidative stress (7-10). Elevated levels of ROS are associated with the degree of trauma in surgery and oxidative stress can lead to chronic inflammation by activation of several transcription factors (11-13). Arsalani-Zadeh et al. reviewed the published studies of oxidative stress in laparoscopic, and open surgery (14). They found that the oxidative stress response to surgery depends on the degree of trauma, and the reduction of surgical trauma 
by laparoscopy seems to diminish the stress response compared to open surgery. To our knowledge, the assessment of GPX1 response to surgical trauma in laparotomy with rectus sheath block (RSB) analgesia has not been studied in a placebo-controlled, randomised trial.

The aim of the study was to evaluate whether the overall satisfaction, as measured by the numeric rating scale (NRS), with post-surgery RSB analgesia was associated with the plasma GPX1 level. The second end-point of the study was to evaluate the differences in GPX1 levels in patients with and without RSB analgesia, with special emphasis on benign or malignant disease status.

\section{Patients and Methods}

The study was approved by the Ethics Committee of Kuopio University Hospital District, Finland (DNRO 120/2011, November 11, 2011), and registered in the EudraCT database (EudraCT number 2011-005136-25) and in the ClinicalTrials.gov database (ClinicalTrials.gov Identifier: NCT02869841) and was conducted in accordance with the Declaration of Helsinki. Participants gave their written consent after receiving verbal and written information. Operations were carried out at Kuopio University Hospital, Kuopio between 2012 and 2015. The CONSORT flowchart of the study is presented in Figure 1. The study design was a prospective, randomised, clinical trial with four parallel groups. Patients undergoing midline laparotomy were randomized into a placebo group or into one of the three active RSB analgesia groups: single-dose, repeated-dose or continuous infusion. Each study patient had an intravenous oxycodone pump as the patientcontrolled analgesia. The randomization list was generated by computer (www.randomization.com), a sealed-envelope method was used for blinding, and randomization was performed preoperatively.

The primary outcome measures were the plasma level of highsensitivity C-reactive protein (hs-CRP) and GPX1 measured in the placebo versus three active groups at three time points with highsensitivity assays.

All RSB procedures were performed by an experienced surgeon in the operating room before wound closure. Bilateral RSB catheters were placed with completely aseptic technique into the 'railway-like line' in the rectus sheath between the rectus muscle and the posterior rectus sheath in the lateral third of the sheath. The correct position of catheters was confirmed and $20 \mathrm{ml}$ levobupivacaine was injected to separate the planes and achieve hydrodissection for placement of the catheters. In the blocks, including the single-dose block, bilateral rectus sheath catheters were used (InfiltraLong Pajunk, Geisingen, Germany). In the single-dose group, the block was performed at the end of surgery by injecting $20 \mathrm{ml}$ levobupivacaine $(1.25 \mathrm{mg} / \mathrm{ml})$ (Chirocaine, AbbVie, Espoo, Finland) into both catheters, with a total dose of $50 \mathrm{mg}$ levobupivacaine. In the repeated-dose group, a similar starting injection was performed, and after that $10 \mathrm{ml}$ of levobupivacaine $(1.25 \mathrm{mg} / \mathrm{ml})$ for both catheters were infused, a total dose of two injections $25 \mathrm{mg}$, was repeated every $4 \mathrm{~h}$ for the first $48 \mathrm{~h}$. In the continuous infusion group, the $20+20 \mathrm{ml}$ block was performed as described above and continued with bilateral rectus sheath catheters, with infusion of $5 \mathrm{ml} / \mathrm{h}$ of levobupivacaine
$1.25 \mathrm{mg} / \mathrm{ml}$ for each (a total dose of $12.5 \mathrm{mg} / \mathrm{h}$ ) with ambulatory infusion pumps (Autofuser pumps; Acemedical, Seoul, Korea). The patients in the placebo group had no RSB catheters inserted; however, they were blinded using similar wound dressing to the patients in the active groups.

The exclusion criteria included: high body mass index (BMI; $\geq 35 \mathrm{~kg} / \mathrm{m}^{2}$ ), age under 18 or 80 years or more, pregnancy, repeat operations during the same hospital visit, history of drug or narcotics abuse, and earlier allergic reactions to local anaesthetics and contraindications to oxycodone. All study patients were informed by the investigator about different postoperative analgesia methods before they gave their written consent.

EDTA-blood samples were taken at three time points: before (PRE), immediately after (POP1) and $24 \mathrm{~h}$ (POP2) after surgery and centrifuged at $1000 \times \mathrm{g}$ for $15 \mathrm{~min}$. Plasma was separated and stored frozen at $-70^{\circ} \mathrm{C}$ until analyzed. The plasma GPX1 assays were performed using sandwich-type enzyme-linked immunosorbent assay (ELISA) from BioVendor GPX1 ELISA Kit (Brno, Czech Republic). Plasma hs-CRP was analyzed with a Cobas 6000analyzer (Hitachi, Tokyo, Japan) using the method by Roche Diagnostics (Mannheim, Germany).

The overall satisfaction and opinion on the success of the analgesic procedure were scored on a 11-point NRS at $24 \mathrm{~h}$ following surgery (NRS $0=$ completely dissatisfied; $10=$ fully satisfied).

The data were analyzed with IBM SPSS Statistics 22.0 (IBM Corp., Armonk, NY, USA). Differences in baseline characteristics between groups were tested by Fisher exact test; for continuous variables, analysis of variance (ANOVA) was performed. Group differences at the three time points were tested by Mann-Whitney $U$-test and Kruskall-Wallis test. The results of the laboratory measurements are presented as medians with interquartile range as distributions were right-skewed. A two-sided $p$-value of less than 0.05 was considered statistically significant. The results of the individual GPX1 values for patients with benign disease and those with cancer patients were assessed by Spearman's correlation coefficient.

\section{Results}

The demographics and the perioperative data were similar in the four study groups, but the BMI and the weight were higher in the patients in the placebo group and in the singledose group than in the two other study groups (Table I).

At baseline, the hs-CRP median values were similar in the placebo group and the three active groups, and the median values increased in all four study groups postoperatively (Table II). However, the elevation of the hsCRP median values $24 \mathrm{~h}$ postoperatively was slightly smaller in the patients of three active groups combined (RSB combined, Table III) than in the hs-CRP in the placebo group (Table II).

There was no statistical significant correlation in the individual NRS values between patients with benign disease and those with cancer $(\mathrm{r}=0.11, p=0.950)$, nor between the individual NRS values and plasma levels of GPX1 $(r=0.170$, $p=0.360$ ). 


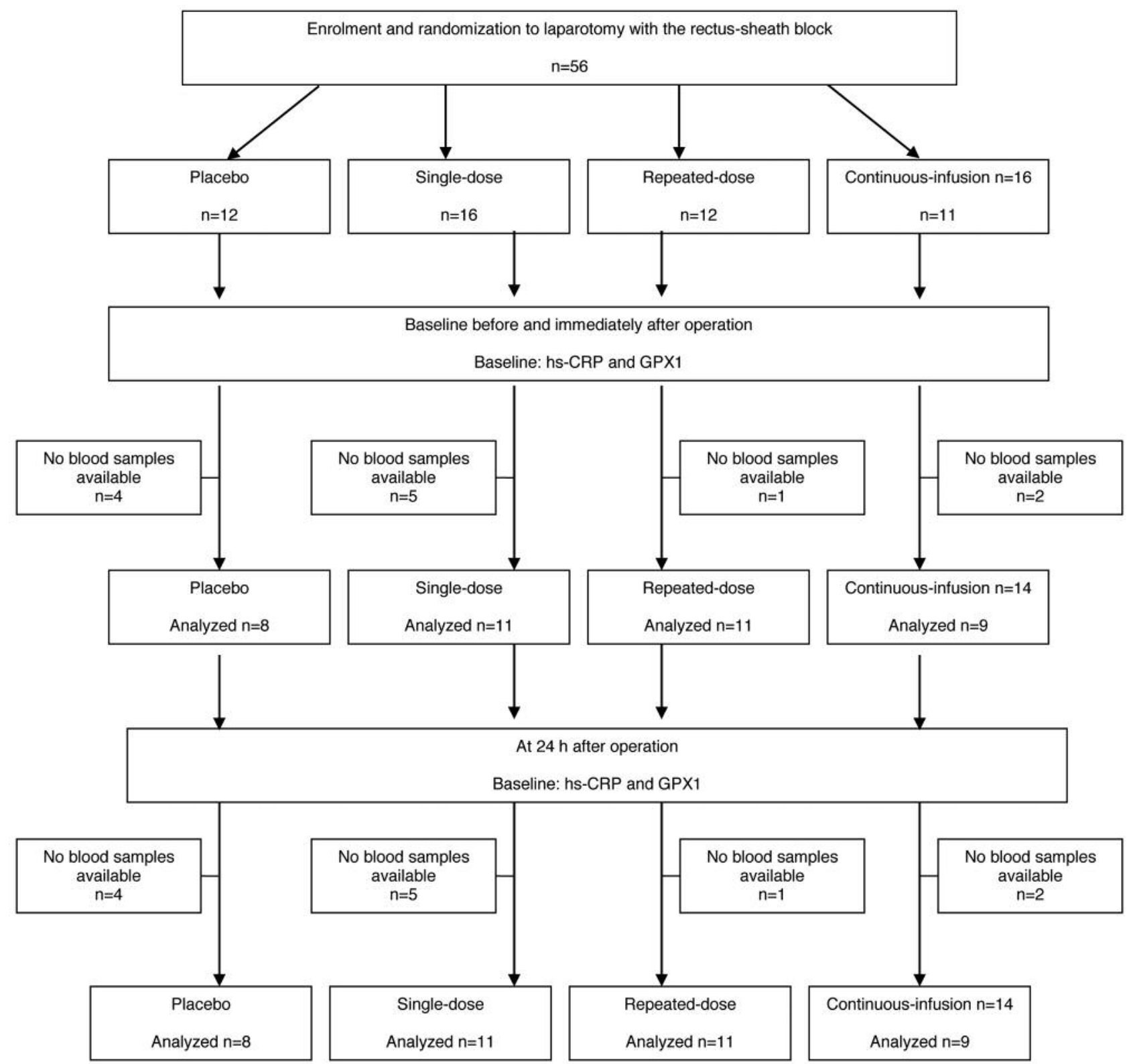

Figure 1. CONSORT figure of the study design.

Plasma levels of GPX1 decreased postoperatively in all four groups (POP1 and POP2, Table II). The patients in the single-dose and repeated-dose groups had significantly lower GPX1 levels $24 \mathrm{~h}$ after surgery (POP2) compared to the three other groups separately $(p=0.032$, Table II). No differences were detected in the GPX1 values preoperatively and immediately after surgery between the placebo and the three active groups combined (Table III).

The median and interquartile range levels of hs-CRP and GPX1 are shown in Table IV. There was no statisticaly significant difference in the median preoperative hs-CRP values between patients with benign disease and those with cancer. However, there was a trend for greater elevation of the median hs-CRP value postoperatively in the cancer group (Table IV). The median (interquartile range) plasma levels of GPX1 was significantly lower preoperatively in the cancer group than in those with benign disease $(p=0.006$, Table IV). Cancer diagnosis was associated with lower individual plasma GPX1 values immediately after surgery $(\mathrm{r}=-0.42, p=0.004$, Figure 2); the patients with cancer appeared to have significantly lower plasma GPX1 values. 
Table I. Baseline demographic characteristics and surgical data for the placebo group and the three active groups; single-dose, repeated-dose and continuous infusion rectus sheath block analgesia groups. Data are mean \pm standard deviation or number of cases.

\begin{tabular}{|c|c|c|c|c|c|}
\hline Variable & Placebo $(n=8)$ & Single $(n=11)$ & Repeated $(n=11)$ & Continuous $(n=14)$ & $p$-Value \\
\hline Age (years) & $62.6 \pm 14.3$ & $60.8 \pm 12.6$ & $63.3 \pm 10.8$ & $58.0 \pm 10.1$ & 0.74 \\
\hline Gender: Male/female (n) & $4 / 4$ & $4 / 7$ & $2 / 9$ & $2 / 12$ & 0.22 \\
\hline Height $(\mathrm{cm})$ & $166.6 \pm 8.6$ & $168.4 \pm 7.9$ & $165.7 \pm 7.2$ & $164.3 \pm 6.6$ & 0.62 \\
\hline Weight (kg) & $78.6 \pm 11.8$ & $83.7 \pm 12.8$ & $67.8 \pm 13.7$ & $68.8 \pm 10.6$ & 0.007 \\
\hline $\operatorname{BMI}\left(\mathrm{kg} / \mathrm{m}^{2}\right)$ & $28.3 \pm 3.8$ & $29.6 \pm 4.4$ & $24.6 \pm 4.3$ & $25.7 \pm 4.9$ & 0.03 \\
\hline Time in the operating room (min) & $229.4 \pm 113.4$ & $274.9 \pm 148.4$ & $235.7 \pm 112.0$ & $279.7 \pm 178.5$ & 0.85 \\
\hline Operative time $(\mathrm{min})$ & $209.6 \pm 141.2$ & $221.8 \pm 156.4$ & $154.4 \pm 95.0$ & $253.3 \pm 168.9$ & 0.55 \\
\hline Perioperative bleed (ml) & $696 \pm 741$ & $822 \pm 906$ & $697 \pm 967$ & $1340 \pm 928$ & 0.31 \\
\hline $\operatorname{ASA} 1 / 2 / 3 / 4(n)$ & $0 / 6 / 2 / 0$ & $0 / 7 / 3 / 1$ & $0 / 5 / 6 / 0$ & $2 / 7 / 5 / 0$ & 0.43 \\
\hline Length of the skin incision $(\mathrm{mm})$ & $27.2 \pm 6.6$ & $24.4 \pm 7.8$ & $24.2 \pm 7.9$ & $29.7 \pm 7.3$ & 0.31 \\
\hline Type of disease: benign/GI-cancer/Gyn-cancer/other $\#$ & $1 / 3 / 3 / 1$ & $3 / 2 / 4 / 2$ & $5 / 2 / 4 / 0$ & $6 / 2 / 6 / 0$ & 0.32 \\
\hline
\end{tabular}

BMI: Body mass index; ASA: American Society of Anesthesiologists physical status score. ${ }^{\# B e n i g n}$ disease $\mathrm{n}=15$, gastrointestinal tract cancer $\mathrm{n}=9$, gynecological cancer $n=17$, other malignancy $n=3$.

Table II. Plasma high-sensitivity C-reactive protein (hs-CRP) and glutathione peroxidase (GPX1) concentrations in the placebo group and the three active groups; single-dose, repeated-dose and continuous infusion rectus sheath block analgesia groups measured at three time points: before (PRE), immediately after (POP1) and $24 \mathrm{~h}$ after (POP2) surgery. Median (interquartile range) values are shown.

\begin{tabular}{|c|c|c|c|c|c|}
\hline Marker & Placebo & Single & Repeated & Continuous & $p$-Value \\
\hline \multicolumn{6}{|c|}{ hs-CRP (mg/l) } \\
\hline PRE & $3.48(1.2-13.0)$ & $4.65(0.89-48.23)$ & $1.20(0.59-4.00)$ & $6.45(0.62-73.54)$ & 0.795 \\
\hline POP1 & $8.20(1.6-14.6)$ & $9.18(2.9-83.3)$ & $2.30(0.59-5.13)$ & $4.80(0.65-63.0)$ & 0.319 \\
\hline POP2 & $311.79(111.5-499.0)$ & $189.5(96.3-349.0)$ & $206.50(81.5-284.4)$ & $159.0(79.3-478.9)$ & 0.857 \\
\hline \multicolumn{6}{|c|}{ GPX1 (pg/ml) } \\
\hline PRE & $12.05(8.8-19.2)$ & $10.70(5.4-16.7)$ & $19.17(6.7-22.0)$ & $17.00(9.5-18.9)$ & 0.622 \\
\hline POP1 & $11.9(8.20-36.4)$ & $9.40(6.1-23.0)$ & $15.10(6.5-29.8)$ & $15.10(10.2-20.6)$ & 0.768 \\
\hline POP2 & $10.57(5.8-16.3)$ & $5.13(3.2-9.2)$ & $8.63(5.3-19.2)$ & $12.29(6.7-19.6)$ & 0.032 \\
\hline
\end{tabular}

\section{Discussion}

The primary end-point of our study was to compare plasma concentrations of hs-CRP and GPX1 in patients with midline laparotomy randomized into the placebo group or to one of three active groups: single-dose, repeated-dose and continuous infusion RSB analgesia groups. The main hypothesis of our study was whether the post-surgery placement of the RSB could reduce oxidative stress following surgery in patients with benign disease or cancer. No statistically significant differences were detected in the median GPX1 values between the patients with benign disease and those with cancer postoperatively. However, the statistically significant inverse correlation between the individual plasma GPX1 values and cancer diagnosis might suggest some role for GPX1 in cancer; unfortunately, we have no explanation for this association, therefore its significance should be very carefully interpreted, since the small sample size $(n=44)$ of analysed patients is one limitation of our study due to the specific nature of this study.
This limitation could be taken into account when planning new scientific studies of the role of oxidative stress and analgesia in patients with cancer.

ROS and reactive nitrogen species (RNS) are products of normal cellular activity. However, at high concentrations, ROS and RNS can damage both nuclear and mitochondrial DNA, RNA, lipids and proteins by nitration, oxidation and halogenation reactions, leading to mutations and genomic instability (1, 15-17). ROS and RNS products can also modulate signaling molecules and alter functions of enzymes and proteins involved in carcinogenesis $(1,15-17)$. In order to avoid cell damage, the living cell needs defense mechanisms against free radicals, such as oxygen, hydrogen peroxide and superoxide, the protective functions of which maintain the balance between intracellular reactive species and antioxidant activity. GPX is an established enzyme of antioxidant defense and GPX1 is the cytosolic form of GPX. This GPX selenium-dependent enzyme is responsible for the detoxification of lipid and hydrogen peroxides (18). 


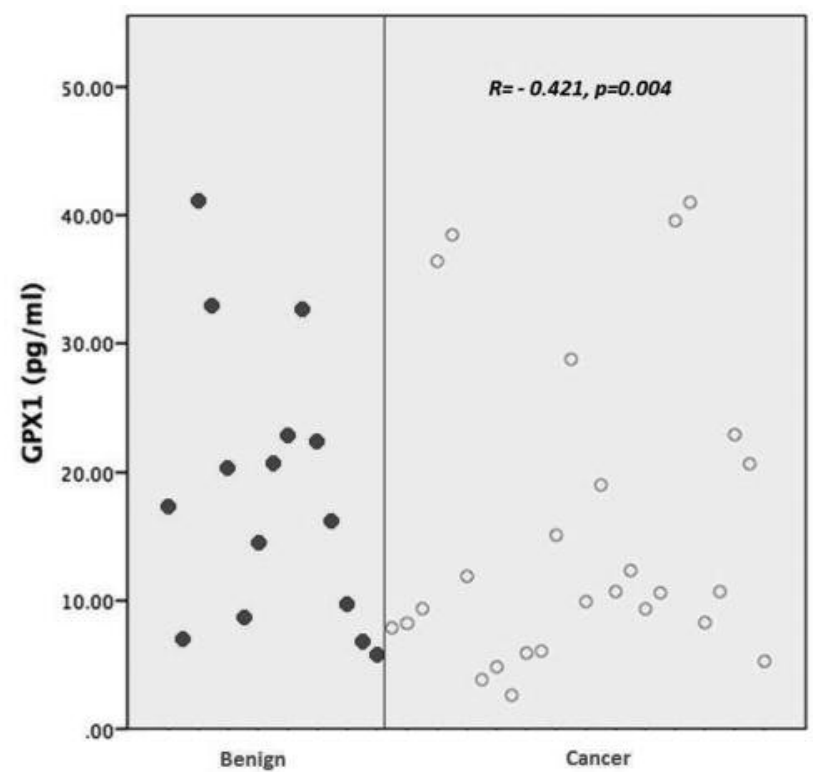

Figure 2. The jitterplot of the individual glutathione peroxidase (GPX1) values immediately after surgery (POP1) according to the diagnosis of the patients as benign disease ( $n=15)$ or cancer $(n=29)$.

We have reported a statistically significant inverse correlation between the individual plasma values for hs-CRP and 8hydroxy-2'-deoxyguanosine $(8-\mathrm{OHdG})$ in patients with benign disease versus those with cancer (9). Although the placement of RSB analgesia does not significantly alter the concentration of $8-\mathrm{OHdG}$ in patients with benign disease or cancer, recent evidence suggests that oxidative stress, inflammation and cancer are related, $(9,10)$ as shown in our study.

We reported that RSB analgesia does not significantly reduce the concentrations of inflammatory response biomarkers interleukin 1 receptor antagonist (IL1RA), IL6, IL8, IL10, IL1 $\beta$ in patients with cancer or benign disease with RSB analgesia randomized into the placebo group or to the three active groups as here (10). However, there was a significant correlation in the satisfaction with pain treatment by NRS with plasma postoperative concentrations of the anti-inflammatorycytokine IL10 and pro-inflammatory cytokine IL1 $\beta$, suggesting that inflammation and pain are related. In the single-dose, repeated-dose and continuous infusion RSB analgesia groups, the RSB analgesia catheters were inserted at the end of surgery, while the patients in the placebo group had no RSB analgesia catheters at all. However, the patients in the placebo group were blinded using similar wound dressing to that for the patients in the active groups. Therefore, it is unlikely that there is a study bias from the placement of the RSB catheters between the placebo group and the three study groups.

Nalkiran et al. studied serum and tissue GPX1 levels in 35 patients with colorectal cancer and found a statistically
Table III. Plasma high-sensitivity C-reactive protein (hs-CRP) and glutathione peroxidase (GPX1) concentrations in the placebo group and in the rectus sheath block (RSB) group (all three active groups combined) measured at three time points: before (PRE), immediately after (POP1) and 24 h after (POP2) surgery. Median (interquartile range) values are shown.

\begin{tabular}{lccc}
\hline Marker & Placebo & RSB & $p$-Value \\
\hline hs-CRP (mg/l) & & & \\
PRE & $3.48(1.21-13.05)$ & $3.30(0.8-36.0)$ & 0.704 \\
POP1 & $8.2(1.6-14.6)$ & $3.90(0.81-53.0)$ & 0.903 \\
POP2 & $311.8(111.5-499.0)$ & $189.6(93.6-302.5)$ & 0.447 \\
GPX1 (pg/ml) & & & \\
PRE & $12.05(8.8-19.2)$ & $15.70(7.7-19.7)$ & 0.927 \\
POP1 & $11.90(8.2-36.4)$ & $11.50(6.9-22.6)$ & 0.469 \\
POP2 & $10.57(5.8-16.3)$ & $8.65(5.2-14.8)$ & 0.703 \\
\hline
\end{tabular}

Table IV. Plasma high-sensitivity C-reactive protein (hs-CRP) and glutathione peroxidase (GPX1) marker concentrations in patients with benign disease $(n=15)$ and those with cancer $(n=29)$ measured at three time points: before (PRE), immediately after (POP1) and $24 \mathrm{~h}$ after $(P O P 2)$ surgery. Median (interquartile range) values are shown.

\begin{tabular}{lccc}
\hline Marker & Benign & Cancer & $p$-Value \\
\hline hs-CRP (mg/l) & & & \\
PRE & $2.20(0.6-3.8)$ & $5.49(1.0-62.8)$ & 0.099 \\
POP1 & $2.50(0.6-6.1)$ & $8.61(0.8-58.8)$ & 0.225 \\
POP2 & $136.0(62.2-254.5)$ & $209.8(100.8-375.0)$ & 0.258 \\
GPX1(pg/ml) & & & \\
PRE & $18.00(12.5-22.0)$ & $10.0(6.3-18.8)$ & 0.006 \\
POP1 & $17.30(8.7-23.1)$ & $10.70(7.4-22.4)$ & 0.250 \\
POP2 & $11.93(6.7-24.7)$ & $8.10(5.2-12.3)$ & 0.120 \\
\hline
\end{tabular}

significant difference in GPX1 expression between normal and cancer tissue. However, they observed a negative correlation between tissue and serum GPX1 levels (19).

In conclusion, the results here suggest that the postsurgical placement of RSB analgesia does not significantly alter the concentration of oxidative stress marker GPX1 following surgery in patients with benign disease or cancer. A new finding with possible clinical relevance is the significant association between the individual plasma GPX1 values and the diagnosis of cancer.

\section{Disclosure and Compliance with Ethical Standards}

The Authors declare that they have no conflict of interest. The Authors alone are responsible for the content and writing of this original article. The study was approved by the Kuopio University Hospital District, Finland (DNRO 120/2011, November 11, 2011), it was registered in the EudraCT database (EudraCT number 2011-005136-25, CONSORT diagram, Figure 1) and in the ClinicalTrials.gov database (ClinicalTrials.gov Identifier: NCT02869841). 


\section{Acknowledgements}

The study was funded by the Heikki, Aino and Aarne Korhonen foundation and the EVO-funding of the Kuopio University Hospital.

\section{References}

1 Bartsch $\mathrm{H}$ and Nair J: Chronic inflammation and oxidative stress in the genesis and perpetuation of cancer: role of lipid peroxidation, DNA damage, and repair. Langenbecks Arch Surg 391: 499-510, 2006.

2 Muller FL, Lustgarten MS, Jang Y, Richardson A and Van Remmen H: Trends in oxidative aging theories. Free Radic Biol Med 43: 477-503, 2007.

3 Reuter S, Gupta SC, Chaturvedi MM and Aggarwal BB: Oxidative stress, inflammation, and cancer: How are they linked? Free Radic Biol Med 49: 1603-1616, 2010.

4 Sedighi O, Makhlough A, Shokrzadeh M and Hoorshad S: Association between plasma selenium and glutathione peroxidase levels and severity of diabetic nephropathy in patients with type two diabetes mellitus. Nephrourol Mon 6: e21355, 2014.

5 Socha K, Kochanowicz J, Karpińska E, Soroczyńska J, Jakoniuk $\mathrm{M}$, Mariak $\mathrm{Z}$ and Borawska $\mathrm{MH}$ : Dietary habits and selenium, glutathione peroxidase and total antioxidant status in the serum of patients with relapsing-remitting multiple sclerosis. Nutrition Journal 13: 62, 2014.

6 Katar M, Ozugurlu AF, Ozyurt $\mathrm{H}$ and Benli I: Evaluation of glutathione peroxidase and superoxide dismutase enzyme polymorphisms in celiac disease patients. Genet Mol Res 13: 1030-1037, 2014.

7 Aspinen S, Kinnunen M, Harju J, Juvonen P, Selander T, Holopainen A, Kokki H, Pulkki K and Eskelinen M: Inflammatory response to surgical trauma in patients with minilaparotomy cholecystectomy versus laparoscopic cholecystectomy: a randomised multicentre study. Scand J Gastroenterol 51: 739-744, 2016.

8 Aspinen S, Harju J, Juvonen P, Selander T, Kokki H, Pulkki K and Eskelinen M: The plasma 8-OHdG levels and oxidative stress following cholecystectomy: a randomised multicentre study of patients with minilaparotomy cholecystectomy versus laparoscopic cholecystectomy. Scand J Gastroenterol 51: 1507-1511, 2016.

9 Purdy M, Kokki M, Anttila M, Aspinen S, Juvonen P, Selander T, Kokki H, Pulkki K and Eskelinen M: Does the post-surgery placement of rectus sheath block analgesia alter the oxidative stress biomarker 8-OHdG concentrations: a randomised trial of patients with cancer and benign disease. Cancer Genomics Proteomics 13: 239-244, 2016.
10 Purdy M, Kokki M, Anttila M, Aspinen S, Juvonen P, Korhonen R, Selander T, Kokki H and Eskelinen M: Does the rectus sheath block analgesia reduce the inflammatory response biomarker IL1ra, IL6, IL8, IL10 and IL1 $\beta$ concentrations following surgery? A randomised clinical trial of patients with cancer and benign disease. Anticancer Res 36: 3005-3011, 2016.

11 Olakowski M, Lampe P, Mekle H and Stefanski L: Changes in activity of antioxidant enzymes in the early period after classical and laparoscopic cholecystectomy. Wiad Lek 50: 213-217, 1997 (in Polish).

12 Seven R, Seven A, Erbil Y, Mercan S and Burçak G: Lipid peroxidation and antioxidant state after laparoscopic and open cholecystectomy. Eur J Surg 165: 871-874, 1999.

13 Pappas-Gogos G, Tellis C, Lasithiotakis K, Tselepis AD, Tsimogiannis K, Tsimoyiannis E, Chalkiadakis G and Chrysos E: Oxidative stress markers in laparoscopic versus open colectomy for cancer: a double-blind randomized study. Surg End 27: 2357-2365, 2013.

14 Arsalani-Zadeh R, Ullah S, Khan S and MacFie J: Oxidative stress in laparoscopic versus open abdominal surgery: A systematic review. J Surg Res 169: 59-68, 2011.

15 Evans MD, Dizdaroglu M and Cooke MS: Oxidative DNA damage and disease: induction, repair and significance. Mutat Res 567: 1-61, 2004.

16 Valko M, Leibfritz D, Moncol J, Cronin MT, Mazur M and Telser $\mathrm{J}$ : Free radicals and antioxidants in normal physiological functions and human disease. Int J Biochem Cell Biol 39: 44-84, 2007.

17 Valavanidis A, Vlachogianni T and Fiotakis C: 8-hydroxy-2'deoxyguanosine (8-OHdG). A critical biomarker of oxidative stress and carcinogenesis. J Environ Sci Health Part C 27: 120139, 2009.

18 Artur JR: The glutathione peroxidases. Cell Mol Life Sci 57: 1825-1835, 2000.

19 Nalkiran I, Turan S, Arikan S, Kahraman ÖT, Acar L, Yaylim I and Ergen A: Determination of gene expression and serum levels of MnSOD and GPX1 in colorectal cancer. Anticancer Res 35: 255-259, 2015. 